\title{
PERFORMANCE ASSESSMENT AND GENERATOR CONDITION MONITORING SYSTEM
}

\author{
Ms.Sahaya Ezhil Priya Tharshni.P \\ M.E.Communication System \\ Rohini College Of Engineering And Technology \\ Kanyakumari, Tamilnadu, India
}

\begin{abstract}
Wind turbines (WTs) are usually operated in harsher environment and, therefore, have relatively higher failure rates. The faults in WTs can be classified into two categories. wear-out failures and temporary random faults. Wear-out failures are long-term and permanent events. Repairing or replacing a failed component needs additional costs and results in a loss of energy production. Data-driven approaches have gained increasing interests in the fault detection of wind turbines (WTs) due to the difficulty in system modeling and the availability of sensor data. A new fault detector based on a recently developed unsupervised learning method, denoising auto encoder (DAE), which offers the learning of robust nonlinear representations from data against noise and input fluctuation. Temporary random faults can usually be cleared by temporarily shutting down and restarting the components with faults or the WTs. In addition, we apply the sliding window technique to consider temporal information inherent in time series data by including the current and past information within a small time window. Using the information obtained from the condition monitoring process, fault diagnosis can be performed to detect, locate, and identify occurring faults and monitor the development of the faults from defects into failures; and prognosis can be performed to predict the development of a defect into a failure, when the failure occurs, and the remaining useful life of the WT component with the defect. Fault diagnosis and prognosis are important extensions of condition monitoring. Based on the diagnostic and prognostic information, the appropriate maintenance strategy can be taken to minimize the maintenance cost, reduce WT downtime, and improve WT reliability and lifespan.
\end{abstract}

Keywords - Wind Turbines(Wts), Denoising Auto Encoder (DAE), Fault Detection

\section{INTRODUCTION}

Wind turbine condition monitoring technologies have been widely adopted in recent years to provide diagnostic information on the health condition of various wind turbine

\author{
Mr. P.Benesh Selva Nesan, M.E., \\ Assistant Professor \\ Rohini College Of Engineering And Technology \\ Kanyakumari, Tamilnadu, India
}

components and subsystems, which allows maintenance to be scheduled and taken before a failure or a critical malfunction occurs. Compared with offline condition monitoring techniques which require the wind turbine to be taken out of service to allow inspection by maintenance personnel, online condition monitoring enjoys the benefit of no interruption on the wind turbine operation and provides a deeper insight into the condition of wind turbine components and subsystems.

The online condition monitoring systems (CMSs) can be classified according to the type of sensors used (e.g. vibration, acoustic, temperature, etc.) or the method of data transmission (e.g., wired or wireless). Most commercially available wind turbine drive train CMSs use vibration signals because most drive train faults and defects will excite new vibration modes or change the existing vibration modes of the drive trains. The sensors and data acquisition devices used in vibration-based CMSs have high costs. Moreover, the performance of vibration-based CMS depends on the locations of vibration sensors. Recently, wind turbine generator current signals have been used successfully for fault diagnosis of wind turbine blades, shaft, bearings, and gearboxes. Compared to vibration signals, the use of generator current signals for drive train condition monitoring has the advantage of lower cost, nonintrusive, independence of sensor locations. In, a comparative study on vibration-and current-based approaches was conducted; the effectiveness of a current-based method for wind turbine gearbox fault diagnosis was validated; and it concluded that current signals were less sensitive to environment noise.

Bratcu et al., (2008) proposed an as exhaustive as possible review of the control methods dedicated to optimizing the dynamic behavior of variable speed wind energy conversion systems (WECS) from various viewpoints. Different performance criteria can be defined for WECS - e.g., the energy optimization can be achieved by operating the system at variable speed. The exploitation experience and grid-integration of WECS has presently concluded the necessity of solving multi-purpose optimization problems, which require advanced control approaches being employed. Some of the most pertinent approaches belonging to this trend 


\section{International Journal of Engineering Applied Sciences and Technology, 2020 Vol. 4, Issue 10, ISSN No. 2455-2143, Pages 405-410 Published Online February 2020 in IJEAST (http://www.ijeast.com)}

and implemented by variable-speed operation are overviewed and assessed here, that allows for suggesting future research issues and perspectives. 13 With rapid development of wind power technologies and significant growth of wind power capacity installed worldwide, various wind turbine concepts have been developed.

The wind energy conversion system is demanded to be more cost-competitive, so that comparisons of different wind generator systems are necessary. An overview of different wind generator systems and their comparisons are presented Li \& Chen (2008). First, the contemporary wind turbines are classified with respect to both their control features and drive train types, and their strengths and weaknesses are described. The promising permanent magnet generator types are also investigated. Then, the quantitative comparison and market penetration of different wind generator systems are presented. Finally, the developing trends of wind generator systems and appropriate comparison criteria are discussed. It is shown that variable speed concepts with power electronics will continue to dominate and be very promising technologies for large wind farms.

Masoud Barakat et al., (2009) have focused on maximum wind power extraction for a WECS composed of a wind turbine, a squirrel-cage induction generator, and a MC. At a given wind velocity, the mechanical power available from a wind turbine is a function of its shaft speed. In order to track maximum power, the MC adjusts the induction generator terminal frequency, and thus, the turbine shaft speed. The MC also adjusts the reactive power transfer at the grid interface toward voltage regulation or power factor correction. A Maximum Power Point Tracking (MPPT) algorithm is included in the control system. Conclusions about the effectiveness of the proposed scheme are supported by analysis and simulation results.

Raguraman et al., (2013) developed an ANN and an MLR model to predict the overall heat transfer coefficient (OHTC) of coal slurry in an agitated vessel used in coal gasification. Arnaud et al (2013) performed the validation of the reconfigurable digital controller for the Wind Energy Conversion Systems (WECS) with Doubly Fed Induction Generator (DFIG) topology using a Hardware-in-the-Loop (HIL) reconfigurable platform including a Field Programmable Gate Array (FPGA) chip. Antonio et al (2013) presented an efficient hardware implementation of configurable circuits as add-in modules for existing fuzzy hardware in FPGA or application-specific integrated circuit (ASIC) for the realization of diverse fuzzy t-norm and $\mathrm{t}$ conorm operations. Pawel szcze'sniak et al., (2013) have done a comparative study on drive system with a permanent magnet synchronous moto fed by a MC and a voltage source inverter with diode rectifier stage. A rotor-oriented vector control is implemented in the drive control. SVM techniques are used for both converters in the output current modulation process.
Initial simulation test results and comparison are presented. The advantages of both solutions are indicated. Special attention is given to the drive control in the low speed range. The main aim of this article is to present drive systems with a $\mathrm{MC}$ as an interesting alternative solution for automation systems for precision control of speed and position.

In the traditional CMSs for electric power system assets, condition monitoring data are mostly transmitted through wired communication channels, which require installation of dedicated communication cables and regular maintenance. Compared to wired communication systems, wireless communication systems, such as wireless sensor network (WSN), have the advantages of easier installation and lower capital, installation, and maintenance costs and, therefore, provide an alternative and promising technology for transmitting condition monitoring data to enhance the reliability of the electric power system assets. In this paper, WSN is adopted for collect the data received from a WSN can be corrupted due to malfunctions of sensors or sensor nodes or interference in the communication channels, which will reduce the accuracy of condition monitoring and fault diagnosis or even lead to false fault diagnosis. Fault-tolerant protocols have been used to verify and correct corrupted data due to interference in communication channels.

The rest of the paper is organized as follows. Proposed algorithm is explained in section II. Experimental results are presented in section III. Concluding remarks are given in section IV.

\section{PROPOSED AlgORITHM}

Wind turbines (WTs) are complex aero-electromechanical energy systems, which consist of hundreds of components and subsystems, including rotor hub, blades, gearbox, generator, power electronics, etc. They are exposed to variable weather conditions and harsh environments and therefore are prone to various faults/failures of sensors, actuators, and components. The unexpected failures and unscheduled maintenance result in high maintenance and operation (O\&M) costs, which are more significant for offshore WTs due to their inaccessibility and harsher operating environments. In order to reduce O\&M costs and improve the availability of WTs, it is highly desirable to develop fault detection systems to provide early warnings of imminent faults, thus helping avoid secondary damages and catastrophic accidents as well as enabling better maintenance planning and logistics.Numerous studies of WT fault detection and diagnosis have been reported in the literature, which could be categorized as model-based approaches and data-driven approaches. Model-based approaches mainly rely on an accurate mathematical model of the WT and its subsystems, including pitch system, drivetrain, and generator, etc., and typically include observer based techniques, Kalman filter and estimators, and parity equations. 


\section{International Journal of Engineering Applied Sciences and Technology, 2020 \\ Vol. 4, Issue 10, ISSN No. 2455-2143, Pages 405-410 \\ Published Online February 2020 in IJEAST (http://www.ijeast.com)}

However, in practice, model-based approaches often fail to work due to the difficulty in modeling multiple coupling in system parameters and unexpected disturbances, which are common especially in large utility-scale WTs.

Wang et al., (2009) write when the wind passes through the turbine, the rotating blades will absorb some amount of wind energy and cast wind in the downwind direction, increasing wind turbulence and the reduction of the wind speed, which is called the wake effect. After that, the wind speed would be gradually recovered, and the recovered value of the wind speed is related to the distance between the upstream and the downstream wind turbines. Therefore, if wind turbines were too dense, the wind speed is unable to adequately recover when reaching the downstream wind turbine, which reduces or stops the output of the downstream wind turbine. In this case, the benefit of power generation is smaller and the investment cost of average unit output is larger. On the contrary, if wind turbines in wind farm were too scattered, the total installed capacity of the whole wind farm is very small, and the investment cost of average unit capacity and the operation maintenance fee are, obviously, high. Therefore, according to the specific condition of the local wind energy of wind farm, once the capacity of wind turbine is selected, the number of the wind turbines and corresponding placement scheme is most important to improve the economy of the wind farm.

Zhang et al., (2007) layout of wind turbines in the wind farm is about optimization of technical, economic, legal, environmental and social aspects. A general conclusion can be drawn with minimum one year data of wind speeds and direction. As each wind turbine will slow down the wind leaving it, the space between turbines should be as far as possible in the prevailing direction. Here the wind rose becomes extremely useful. There should be fewer obstacles and smoother terrain in prevailing wind directions. Based on experience wind turbines are usually spaced between 5 to 9 rotor diameters apart in the prevailing wind direction and between 3 to 5 rotor diameters apart in the direction perpendicular to the prevailing winds.

Mohamed et al., (2012) write on hills wind speeds are higher than in the surrounding area. As a result, wind turbines are commonly placed on hills or ridges overlooking the surrounding landscape. If the hill is steep or has uneven surface, significant amounts of turbulence occur and the advantage of higher wind speeds may be lost. The air becomes compressed on the windy side of hills or mountains, and its speed increases considerably between obstacles. This is known as "the tunnel effect". Placing a wind turbine in a natural tunnel is one way of obtaining higher power outputs. To obtain a good tunnel effect the tunnel should be "softly" embedded in the landscape. Rough and uneven hills produce wind turbulence that may negate the wind speed advantage; the changing winds may inflict a lot of useless tear and wear on the wind turbine.

In contrast, data-driven approaches do not require physical or accurate mathematical models but directly use the measured sensor data to infer the fault detection system. A distinct feature of data-driven approaches is that no prior information about the system is necessary, which is more suitable for such complex WT systems with highly nonlinear dynamics and uncertainty. On the other hand, most modern WTs have installed standard supervisory control and data acquisition systems to collect operational data and status data.Normally, sensory data measured from WTs are multivariate in nature and highly correlated due to the interaction and dependence between different subsystems in a WT, which is similar to many other industrial processes or systems like semiconductor process, gas turbines, electric vehicles, etc. Indeed, the health of a component or subsystem of a WT is related to multiple sensor variables. Usually, when a fault occurs in a certain component, multiple sensor signals may change simultaneously, and also correlations of multivariate data might be affected. Therefore, it is better to monitor the relations in multiple variables to detect and identify faults well. This paper focuses on the multivariate correlations modelling and the reconstruction error based fault detection approach. Recently, traditional multivariate process monitoring approaches like principal component analysis (PCA) have been used to detect sensor and gearbox faults in WTs. However, PCA is based on the assumption of linear process behavior which may not be suitable for WT systems. To overcome its shortcomings, the auto encoder neural network and the newly developed deep auto encoder model have been applied to WT monitoring and fault detection, and yield better performance.However, some challenges in fault detection of WTs still remain. One major challenge lies in their nonlinearity, unknown disturbances as well as significant measurement noise. Practically, WTs are driven stochastically by the wind and subject to various disturbances, leading to more noise and variations of data, and increasing the difficulty to discover abnormal pattern from noisy multivariate data. 


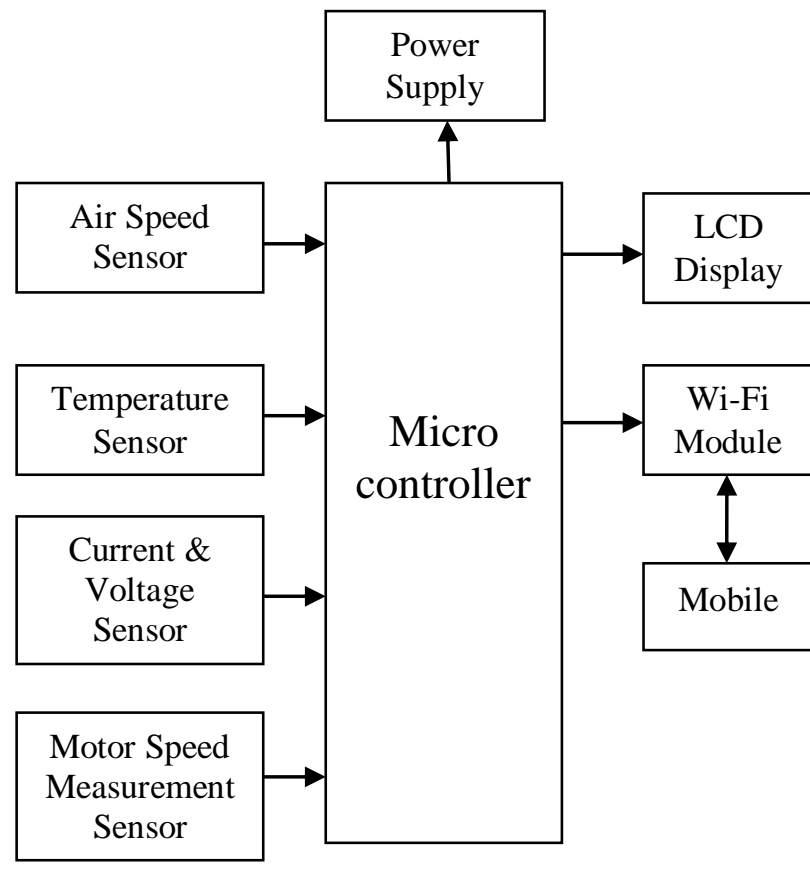

Fig. 1.Block Diagram Of Proposd System

In this case, the existing traditional PCA-based and AEbased approaches will lead to biased monitoring results due to their sensitivity to the disturbances. Another challenge is the temporal dependency in multivariate time series data, which is often ignored by many static monitoring approaches assuming that the current sampling data are statistically independent of the previous observation. In the field of industrial process monitoring, to address the above similar issue, one common strategy is to incorporate previous information into current observation vector to account for the serial correlation of the data, as in dynamic PCA (DPCA), dynamic partial least square (DPLS), and other similar approaches. This strategy is also adopted in our approach to incorporate temporal information in sensor data of WTs. We first developed a fault detector based on a recently emerged algorithm, denoising auto encoder (DAE), to address the first challenge mentioned above. Recent studies in deep learning have shown that DAE can learn more robust representation from corrupted data, have improved generalization capability, and produce the state-ofthe-art performance on many challenging feature learning tasks, such as image classification, object tracking, saliency detection, etc. Motivated by the successful applications and the excellent properties of DAE, we adopted DAE to deal with complex multivariate noisy data from WTs. Our goal is to provide a robust signal reconstruction in the case of small perturbations or disturbances presented in sensor data while capturing nonlinear correlations embedded in multivariate data. Meanwhile, in order to incorporate temporal information, we adopted the sliding window technique to include the current and previous information within a small time window. Thus, a sliding window denoising auto encoder approach named SW.

\section{EXPERIMENT AND RESULT}

The Proposed system use Arduino, which make more power efficient and more reliable to use. This system uses microchip wireless digital chips to communicate to other devices, this communication network now as Wi-Fi module. This system has current sensors sense the current in output of the wind turbine generation. The sensor is connected to the controller. The sensor information is displayed in the mobile through Wi-Fi. IR sensor, voltage sensor air speed sensor and Temperature sensor is connected to the arduino. The pitot tube and MPXV7002DP are connected and wired to the Arduino board, the user should take some basic analog readings to test the system and its response. You can do this by blowing into the pitot tube or using a fan. The first thing to note is the baseline value. It should be $1023 / 2=511.5$, however, this value is not an integer - so we can blindly assume either 511 or 512 are the expected baseline values. Now, in my case, the baseline was a bit higher (534), so I implemented an offset average in the setup portion of the Arduino code. The screw terminals of the ASC712 Current Sensor Module board are connected in series with the wind motor. Then connect the VCC, GND and OUT of the ASC712 board to +5V, GND and A0 of Arduino. Connect the "S" and "-" pins of the Voltage Sensor to A0 (Analog Input) and GND of Arduino respectively. Then connect the external voltage pins (voltage to be measured) to the screw terminal (check for polarity).

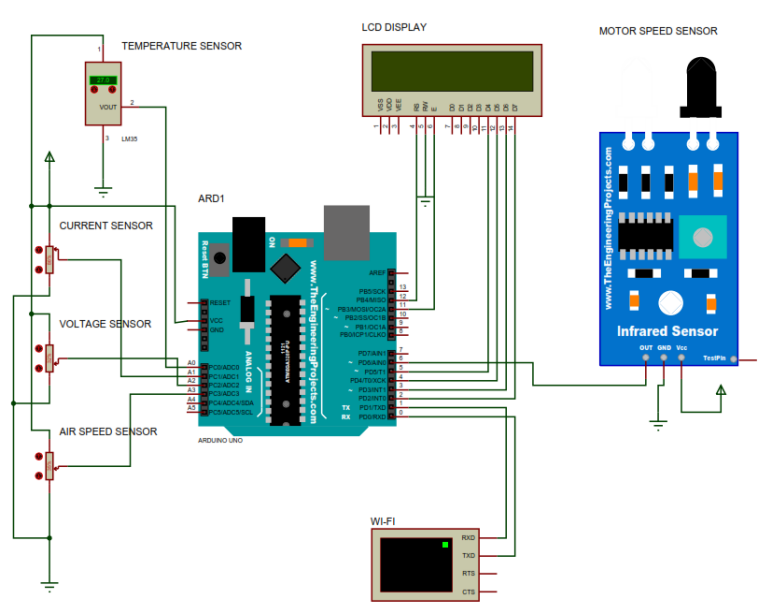

Fig. 2.Circuit Diagram Of Proposd System 


\section{International Journal of Engineering Applied Sciences and Technology, 2020 \\ Vol. 4, Issue 10, ISSN No. 2455-2143, Pages 405-410 \\ Published Online February 2020 in IJEAST (http://www.ijeast.com)}
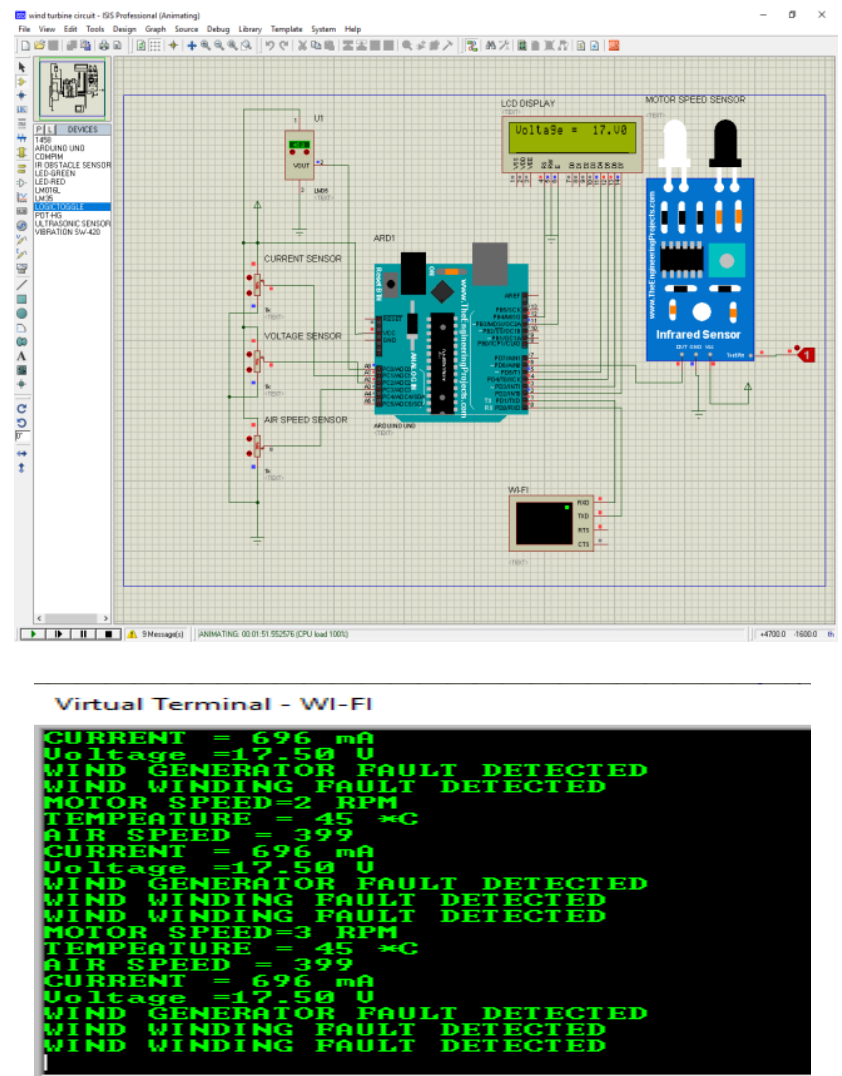

Fig. 3.Simulation Output Of Proposd System

\section{CONCLUSION}

Thus the new multivariate data-driven fault detection approach SW-DAE for WTs. In the proposed approach, we first apply the sliding window on multivariate time series data and then build the DAE model to capture the nonlinear correlations among multiple sensor variables and the temporal dependency of each sensor variable simultaneously. The proposed approach builds the reference model offline using multivariate normal data and then identifies potential faults online by comparing monitoring indicators derived from residuals. Compared with the static approaches (DAE, AE, and PCA) and the extended dynamic approaches (DPCA and SW-AE), our proposed method achieved better fault detection performance in terms of AUC metric in both simulated and real case studies. The results also proved the advantages of DAE in dealing with multivariate noisy data over PCA and the basic AE, especially in capturing nonlinear correlations and providing robust signal reconstruction. More importantly, incorporating the temporal information has greatly improved the fault detection performance, which indicates the importance of temporal dependency in time series data in the design of fault detection algorithms. We are still working on exploring new strategies to incorporate and utilize more complex temporal information.

\section{REFERENCE}

[1] Lapira E., Brisset D., Ardakani H.D., Siegel D., and Lee J. ( 2012). "Wind turbine performance assessment using multi-regime modeling approach," Renewable Energy, vol. 45, (pp. 86-95).

[2] Aghili F., and Su C.Y. (2016). "Robust relative navigation by integration of icp and adaptive kalman filter using laser scanner and imu," IEEE/ASME Transactions on Mechatronics, vol. 21, no. 4, (pp. 2015-2026).

[3] Jiang G., He H., Xie P., and Tang Y. ( 2017).“'Stacked multilevel-denoising autoencoders: A new representation learning approach for wind turbine gearbox fault diagnosis," IEEE Transactions on Instrumentation and Measurement, vol. 66, no. 9, (pp. 2391-2402).

[4] Skrimpas G.A., Sweeney C.W., Marhadi K.S., Jensen B.B., Mijatovic N., and Holbll J. ( 2015). "Employment of kernel methods on wind turbine power performance assessment," IEEE Transactions on Sustainable Energy, vol. 6, no. 3, (pp. 698-706).

[5] Sanchez H., Escobet T., Puig V., and Odgaard P. ( 2015). "Fault diagnosis of an advanced wind turbine benchmark using interval-based arrs and observers," IEEE Transactions on Industrial Electronics, vol. 62, no. 6, (pp. 3783-3793).

[6] Tautz-Weinert J., and Watson S.J. ( 2016). "Using scada data for wind turbine condition monitoring-a review," IET Renewable Power Generation, vol. 11, no. 4, (pp. 382-394).

[7] Park J.Y., Lee J.K., Oh K.Y., and Lee J.S. ( 2014). "Development of a novel power curve monitoring method for wind turbines and its field tests," IEEE Transactions on Energy Conversion, vol. 29, no. 1,( pp. 119-128).

[8] Odgaard P.F., and Stoustrup J. ( 2012). "Fault tolerant control of wind turbines using unknown input observers," IFAC Proceedings Volumes, vol. 45, no. 20, (pp. 313 318).

[9] Odgaard P.F., Stoustrup J., and Kinnaert M. ( 2013). "Fault-tolerant control of wind turbines: A benchmark model," IEEE Transactions on Control Systems Technology, vol. 21, no. 4, (pp. 1168-1182).

[10] Odgaard P.F., and Stoustrup J. (2015). "A benchmark evaluation of fault tolerant wind turbine control concepts," IEEE Transactions on Control Systems Technology, vol. 23, no. 3, (pp. 1221-1228).

[11] Yin S., Wang G., and Karimi H.R. ( 2014). "Data-driven design of robust fault detection system for wind turbines," Mechatronics, vol. 24, no. 4, (pp. 298-306).

[12] Gill S., Stephen B., and Galloway S. (2012). "Wind turbine condition assessment through power curve copula modeling," IEEE Transactions on Sustainable Energy, vol. 3, no. 1, (pp. 94-101). 
[13] Butler S., Ringwood J., and O’Connor F. (2013). "Exploiting scada system data for wind turbine performance monitoring," in Control and fault-tolerant systems (systol), 2013 conference on. IEEE,( pp. 389394).

[14] Qiao W., and Lu D. (2015). "A survey on wind turbine condition monitoring and fault diagnosis part i: Components and subsystems," IEEE Transactions on Industrial Electronics, vol. 62, no. 10, (pp. 6536-6545). 